\title{
Medizinhistorische Dissertationen 1970
}

Doktorarbeiten aus dem Medizinhistorischen Institut der Universität Basel 1970

I. Unter der Leitung von Prof. Dr.med.H.Buess

a) Im Jahre 1970 gedruckte Dissertationen:

P. J. Dubler, Johann Jakob Baader (1810-1879), Arzt und Politiker in Gelterkinden

b) Im Jahre 1970 fertiggestellte Dissertationen:

A. R. Moshfegh, C.S. Haegler (1862-1916)

O. Karamehmedovic, Ernst Tavel (1858-1912)

D. Tetzlaff, Johannes von Miculicz und die Herausbildung der aseptischen Hilfsmittel

II. Unter der Leitung von PD Dr. H.M. Koelbing

a) Im Jahre 1970 gedruckte Dissertationen:

S.ZüLlIG, Luigi Galvani (1737-1798), Der Entdecker der Bioelektrizität

b) Fertig, aber noch nicht gedruckt:

J.Landmann, Diagnostik in der ärztlichen Praxis nach Samuel Gottlieb Vogel (1750-1837)

Doktorarbeiten aus dem Medizinhistorischen Institut der Universität Zürich

Seit November 1969 gedruckte Dissertationen:

101. J. M. Bruttin, Différentes théories sur l'hystérie dans la première moitiê du $19^{e}$ siècle (z M A 66) 1969

102. D.T.Stinson, The Role of Sir William Lawrence in 19 th century English surgery (zMA 67) 1969

103. F.Guggenheim, Bruno Bloch (zma 68) 1969 
104. Th. Achard, Der Physiologe F.Bidder (1810-1894) (zм а 69) 1969

105. U.BuEcK-Rich, Ernst Heinrich Weber und die Hautsinne (zм A 70) 1970

106. R. Kunz, Johann Caspar Lavaters Physiognomielehre im Urteil von zeitgenössischen Ärzten (z м 71) 1970)

107. H. JAPP, Strümpell und die Psychosomatik, 1970

108. N. Egli, Der Prix Montyon de Physiologie expérimentale im 19. Jahrhundert (zMA 72) 1970

109. A. Eichmann, Ismar Boas und die Entwicklung der Gastroenterologie (z м A 73) 1970

110. R.Bischofberger, Jean André Venel (1740-1791) (z ma 74) 1970

111. K. MüLler, Moritz Traube und seine Theorie der Fermente (z м A 75) 1970

112. H.U. Keller, Die letzte Epidemie von Suette Miliaire (1887) (zм A 76) 1970

113. C. Rutz, Die Garrods (z m A 77) 1970)

114. H.R.Stahel, Der weiße Mantel (zм A 78) 1970

115. S. Kessler, Folie à deux (z m A 79) 1970

116. H. WASER, Die Anfänge der Wiederbelebung in der Narkose (zма 80) 1970

117. B.v.Felten, Ignaz Doellingers Vorlesung über Pathologie (zma 81) 1970

118. H.P. Wick. F. H.Erismann (1842-1915), Russischer Hygieniker - Zürcher Stadtrat (zM A 82) 1970 